\title{
In situ TEM observations of reverse dislocation motion upon unloading of tensile-deformed UFG aluminium
}

\author{
F. Mompiou ${ }^{1}$, M. Legros ${ }^{1}$, D. Caillard ${ }^{1}$, H. Mughrabi ${ }^{2}$ \\ ${ }^{1}$ CEMES-CNRS, 29 rue Jeanne Marvig, BP4347, F-31055 TOULOUSE, France. \\ ${ }^{2}$ Department of Materials Science and Engineering, University Erlangen-Nürnberg, \\ Martensstrasse 5, D-91058 Erlangen, Germany. \\ caillard@cemes.fr
}

\begin{abstract}
An in situ transmission electron microscopy study has been performed on ultrafinegrained (UFG) aluminium during tensile loading and unloading in the microyield regime. The goal was to assess the reasons for the unusually large inelastic backflow that had been observed earlier during unloading on UFG material, as compared to that of conventional grain size material. It was noted that in particular edge dislocations emitted by sources within the grains during loading run back into the dislocation sources and disappear during unloading, explaining at least semi-quantitatively the rather large inelastic backflow in UFG material.
\end{abstract}

\section{Introduction}

Bulk ultrafine-grained (UFG) metals produced by equal channel angular pressing (ECAP) have attracted much attention during the last decade because of their extraordinary strength properties [1]. In the present study, the tensile loading and unloading behaviour during microyielding of UFG aluminium specimens was investigated by in situ transmission electron microscopy (TEM). The motivation for this work stems from the observation that UFG copper [2] and aluminium [3] specimens exhibited unusually large inelastic reverse deformations during unloading, whereas conventional grain (CG) size material showed almost completely elastic behaviour during unloading. Figures $1 \mathrm{a}$ and $1 \mathrm{~b}$ show as an example the microyielding loading/unloading behaviours observed on UFG and CG aluminium specimens, respectively [3].

\section{Experimental procedure}

UFG aluminium of commercial purity with a $300 \mathrm{~nm}$ average grain size was obtained by ECAPprocessing with 8 route $B_{c}$ passes, with a rotation of $90^{\circ}$ in the same sense around the specimen axis after every pass (courtesy of Johannes May, Erlangen). Rectangular microsamples, of size $3 \mathrm{~mm} \times 1 \mathrm{~mm}$, were prepared by spark cutting, followed by mechanical and electro-polishing. They were fixed on a Gatan room-temperature straining device, and strained in a 2010HC transmission microscope [4]. The fixation was such that the stress could be released without inducing any compression. The images were recorded by a Megaview III video camera, and analyzed frame by frame. 

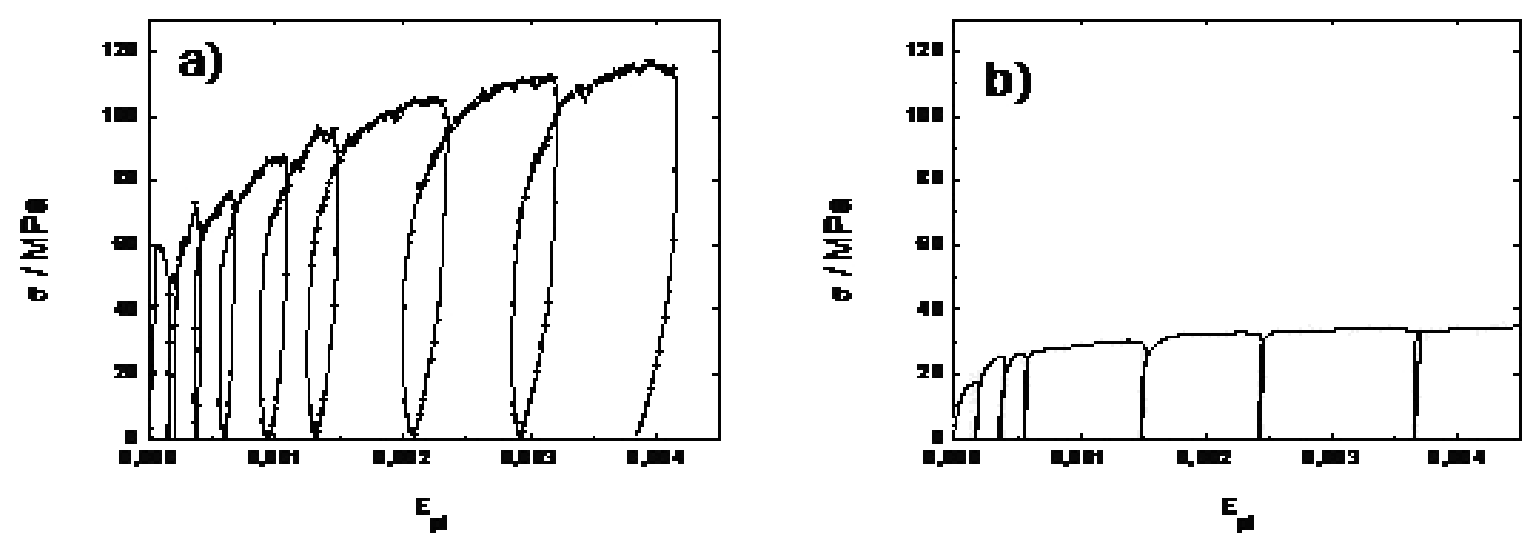

Fig. 1: Tensile microyielding stress-strain curves (plot of stress $\sigma$ versus plastic strain $\varepsilon_{\mathrm{pl}}$ ) with repeated unloadings and reloadings. a) UFG aluminium, as-ECAP. b) CG aluminium. From [3].

\section{Results}

As soon as the stress is applied, sources start to operate in some grains. They all consist of one dislocation segment rotating around a single anchoring point, generally a dislocation junction or a super-jog. This type of source is sometimes referenced as "single-armed source" or "spiral source". Only a few grains deform plastically at yield, presumably those with the largest sizes, the most favourable orientations, and the most appropriate dislocations and anchoring points. All emitted dislocations glide to the neighbouring grain boundaries (GBs), and almost never annihilate inside the grains. Then, more and more grains are deformed till the plastic deformation becomes homogeneous.

In thin foils, the character of the dislocations interacting with GBs is strongly determined by the sample orientation, which can induce different behaviours, as shown in figs. 1 and 2.

Fig. 2 shows a source rotating anticlockwise and emitting 4 dislocation loops in a 600nm grain. The anchoring point $S$ is a super-jog. The slip traces at the foil surface are stair-shaped, which indicates that the dislocations have cross slipped in front of the left GB, as a result of their strong screw component. One consequence of this behaviour is that the 4 dislocations have entered the left GB at different places. Upon unloading, no large reverse dislocation motion could be observed, except for the unbowing of the source dislocation (not shown in the figure).

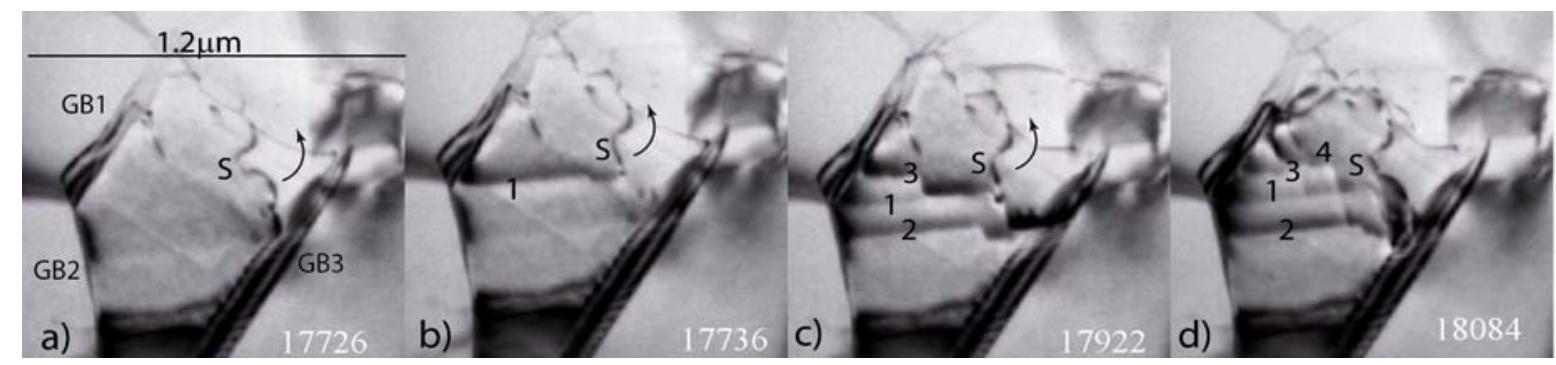

Fig. 2: Single-armed dislocation source (anchoring point S) emitting 4 near-screw dislocation loops. As a result of their cross-slip (see stair-shaped traces, noted 1 to 4), the dislocations enter the left GBs

(GB1 and GB2) at different places. No reverse motion of dislocations (that characterizes inelastic behaviour) has been observed upon subsequent unloading.

Fig. 3 shows another grain containing a single-armed source, anchored in S', rotating anticlockwise, and emitting 5 dislocation loops. At variance from the preceding case, no cross slip takes place in front of the two neighboring GBs, because of the strong edge character of the dislocation segments. Then, all dislocations enter the GBs at almost the same place. Small "pile" ups are formed. In aluminium, this may appear surprising but can be explained by two observations: i) pile ups are not perfect 
because dislocations do not lie in the same exact plane, and ii) the pile-up length is short on account of the small grain size. As shown in what follows, the inelastic reverse dislocation flow effect is large.

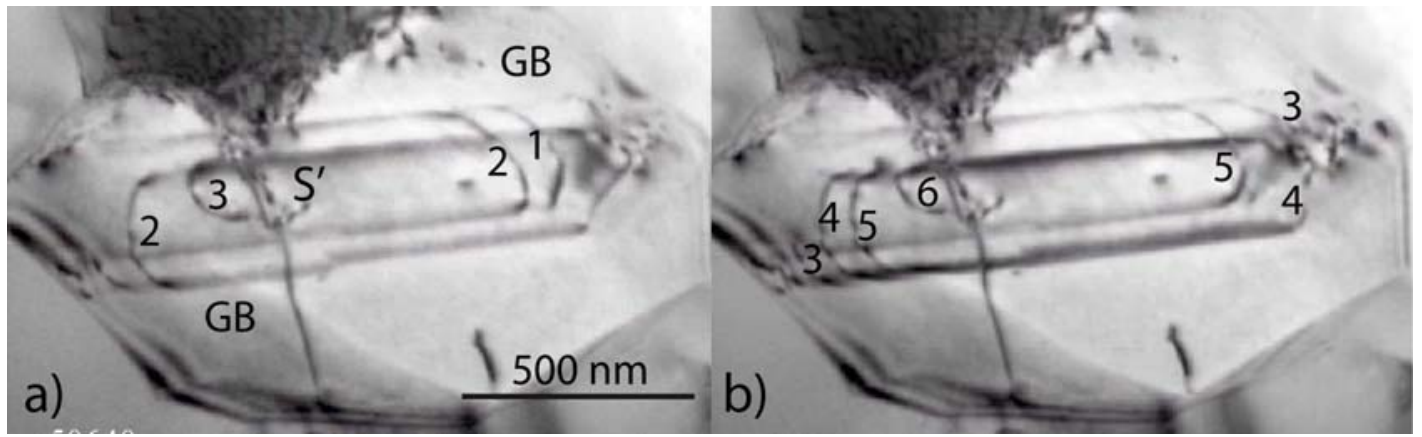

Fig. 3: Dislocation source (anchoring point $S^{\prime}$ ) emitting 6 near-edge dislocation segments. The emitted dislocations form short pile-ups and enter the neighbouring GBs at almost the same place.

Fig. 4 shows the same grain as in fig. 3. On its left side, the source S' emits 2 dislocations which immediately enter the left GB, in (b) and (d). The contrast of the inserted dislocations rapidly vanishes, between (b) and (c), and between (d) and (e). No reverse motion of these dislocations is observed upon unloading, in (e) and (f), although this has occurred at different times of the experiment (see fig. 5). On the right side of the source, a strong broad contrast, noted $\mathrm{C}$, can be seen at the intersection of the active slip plane and the right GB. This contrast results from the cumulated strain contrasts of all previously-inserted dislocations. Dislocation 1 is not completely inserted in the rightside GB, in (b-d), and dislocation 2 is piled up against dislocation 1, in (d), which shows that the broad contrast corresponds to a repulsive elastic stress. Upon unloading, as shown in (e) and (f), this stress is sufficient to extract dislocation 1 from the GB, and to induce the back motion of dislocations 1 and 2 over a fairly large distance.

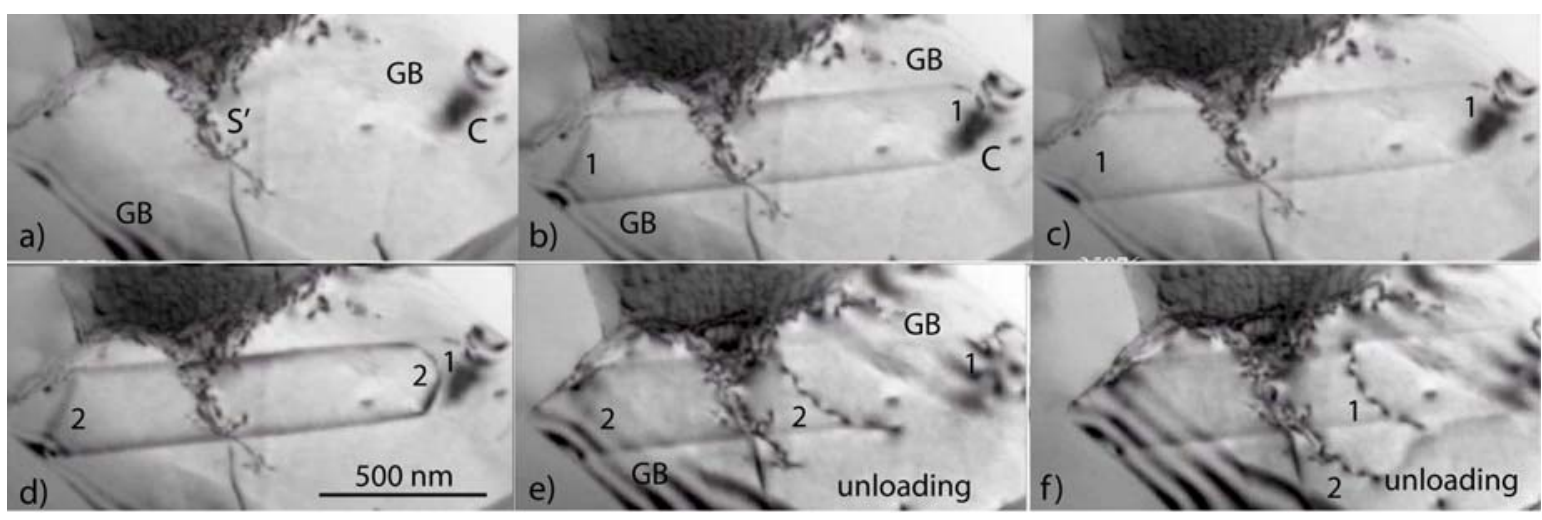

Fig. 4: Inelastic effect in the grain of fig. 2 during loading-unloading. a) The broad contrast $C$ is due to previously inserted dislocations. b) During loading, he first emitted dislocation (1) is completely inserted in the left GB, and partly inserted in the right one. c) The contrast of 1 vanishes in the left GB. d) A second dislocation has been emitted, which is repelled by the GB on the right side. e) After unloading, dislocation 2 moves back on the right side. f) Dislocation 1 is extracted and also moves back (nothing occurs on the left side).

Fig. 5 shows the same behaviour at the left GB. The same source emits a dislocation loop, noted 1, which enters the left GB, as in fig. 4 . The inserted part on the left side is noted $1_{\mathrm{a}}$. This insertion is however incomplete, because one portion noted $1_{b}$ remains out of the GB (fig. 5b). Upon unloading, the portion $1_{b}$ moves back, (fig. $5 \mathrm{c}$ ), and extracts the inserted segment $1_{\mathrm{a}}$ (fig. $5 \mathrm{~d}$ ) till the whole 
dislocation disappears at the source (not shown). This sequence shows that the left GB contains the same repulsive stress field as the right one, although it does not exhibit the same strong broad contrast (note however a distortion in the fringe spacing of the left GB). In other words, repeated insertions at the same GB place generate a strong repulsive stress field, which can sometimes (but not always) appear as a broad TEM contrast.

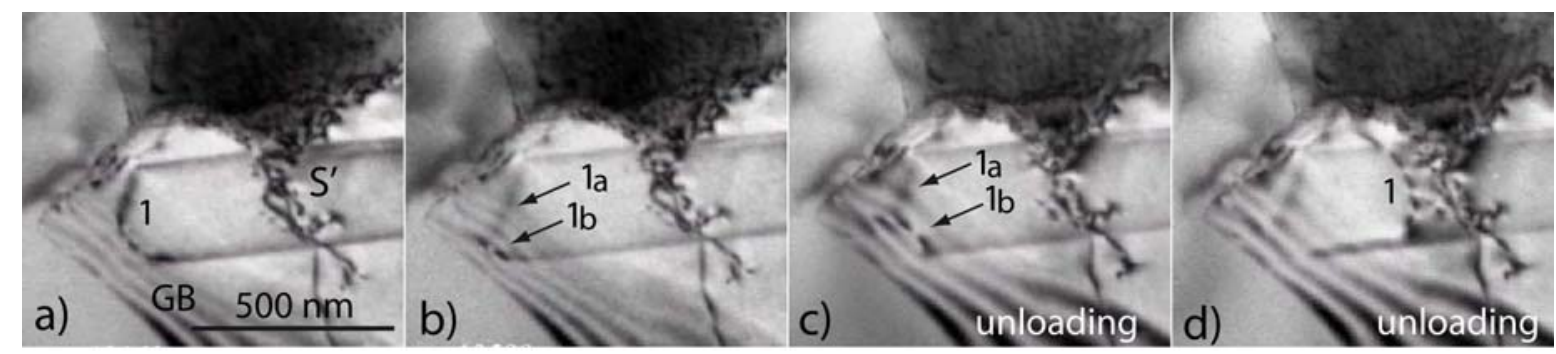

Fig. 5: Same behaviour as in fig. 4, at the left GB. a) After emission of loop 1. b) On the left-side, dislocation 1 is partly inserted in the GB (1a) and partly out of the GB (1b). c) Upon unloading, $1 b$ bows in the back direction. d) Dislocation 1 is completely extracted and moves back to the source.

\section{Discussion and conclusions}

When dislocations that are emitted by sources during loading have a strong edge character, their interaction with GBs shows a strong inelastic reverse motion when the stress is reversed. Using the Orowan law, and considering that 2 dislocations on average move back to the source upon unloading, the inelastic reverse strain in the grain studied is about $5 \times 10^{-4}$. This is equivalent to what has been measured in macroscopic tests, indicating that in this case the inelastic effect is well reproduced in the thin foil.

The internal stress at the origin of the back motion of dislocations originates from the cumulated elastic stress fields of the dislocations inserted previously in the GBs. This explains why the inelastic reverse flow effect is more pronounced for edge dislocations (which all enter the GBs at the same place) than for screw ones (which are more dispersed by cross slip). In the bulk material, the inelastic reverse flow effect should however be present in each grain containing a source, because the emitted dislocation loops always exhibit a near-edge character somewhere at one of the surrounding GBs.

It is noted that the observations made in the earlier mechanical microyielding studies $[2,3]$ and in the present in situ TEM investigation indicate that the dislocations generated during loading disappear almost completely during unloading, implying that there is no net increase of dislocation density after a loading/unloading cycle. This matches nicely the results obtained by Van Swygenhoven's group in an in situ X-ray broadening study on tensile-deformed nanocrystalline nickel [5]. In this work, the Xray peak broadening observed during loading was found to disappear completely during unloading.

\section{References}

[1] Valiev RZ, Islamgaliev RK and Alexandrov IV 2000 Progr. Mater. Sci. 45 103-189.

[2] Höppel HW, Xu C, Kautz M, Barta-Schreiber N, Langdon TG and Mughrabi H 2004 Proc. 2nd Internat. Conference on Nanomaterials by Severe Plastic Deformation Vienna ed M Zehetbauer and RZ Valiev (Weinheim: WileyVCH) 677-683.

[3] Mughrabi H, Höppel HW and Kautz M 2004 Scripta Mater. 51 807-812.

[4] Pettinari F, Couret A, Caillard D, Molénat G, Clément N and Coujou A 2001 J. Microscopy 203 47-56.

[5] Budrovic Z, Van Swygenhoven H, Derlet PM, Van Petegem S and Schmitt B 2004 Science 304 273-276. 